\title{
USO DA GESTÃO DA INFORMAÇÃO E DO CONHECIMENTO NA AVALIAÇÃO DA POLÍTICA PÚBLICA DE ASSISTÊNCIA ESTUDANTIL: ALGUMAS QUESTÕES TEÓRICAS E EMPÍRICAS
}

http://dx.doi.org/10.5902/2318133829803

\author{
Fidel Terenciano \\ Universidade Federal de São Carlos, Brasil. \\ Universidade Católica de Moçambique, Moçambique. \\ António Salião \\ Universidade Católica de Moçambique, Moçambique.
}

\section{Zito Pedro}

Instituto Superior de Gestão, Comércio e Finanças, Moçambique.

\begin{abstract}
Resumo
Neste artigo tem-se por objetivo apresentar questões sobre a política pública de assistência estudantil - Pnae -, e algumas propostas de pesquisa, a partir do uso da informação e do conhecimento no processo de avaliação da implantação desta política pública. A Pnae foi instituída pelo decreto n. 7.234/2010 e visa a possibilitar a permanência e a conclusão dos cursos pelos estudantes em situação de vulnerabilidade socioeconômica. O pressuposto é que para avaliar a gestão afetiva da política é necessário entender o conjunto de estratégias de gestão de informação e conhecimento como importante para compreender o mapeamento dos fluxos de informação e possibilitar a coleta e análise das informações. A metodologia adoptada foi empírica e exploratória e trabalhou-se com a revisão bibliográfica e dados primários sobre a produção e análise na gestão de informação para avaliar as políticas públicas. Os resultados evidenciam que é possível um estudo mais articulado e aprofundado que inclui a gestão de informação e do conhecimento como variável independente na explicação da efetividade e eficácia na implantação da política pública de assistência estudantil.
\end{abstract}

Palavras-chave: gestão da informação e do conhecimento; política pública; assistência estudantil; Pnae. 


\title{
USE OF THE MANAGEMENT OF INFORMATION AND KNOWLEDGE IN THE EVALUATION OF PUBLIC POLICY STUDENT ASSISTENCE: SOME THEORICAL AND EMPIRICAL DISCUSSIONS
}

\begin{abstract}
The article aims to present issues on the public policies of Student Assistance, and brings some research proposals from the use of information and knowledge in the evaluation process of the implementation of public policy. This policy was instituted (PNAES, Decree No. 7234, 2010) which aims to enable the permanence and completion of courses by students in socio-economic vulnerability. The assumption is that to evaluate the affective management policy, you must associate the set of strategies and information and knowledge management are important to understand the mapping of information flows, and enables the collection and analysis of information. The methodology is empirical and exploratory works on the literature review and primary data on production and analysis in information management to evaluate public policies. The results show that a more articulated and in - depth study that includes the information and knowledge management variable is possible as an independent variable in explaining the effectiveness and efficacy in the implementation of the public policy of student assistance.

Key-words: information and knowledge management's; public policy's student assistance; Pnae.
\end{abstract}




\section{Notas introdutórias}

universidade e o papel que desempenha na transmissão de saber não deve
simplesmente basear-se na formação e em habilitar os estudantes a serem
bons profissionais, deve antes de mais, preocupar se em reconstruir a sociedade a partir da transmissão de conhecimentos sobre a mesma realidade. $E$ as políticas públicas educacionais refletem os processos de transformação da própria sociedade como resultante das ações concretas do estado. A educação é, antes de mais, um direito e o dever do Estado proporcionar em melhores condições, pois permite o desenvolvimento humano e social (Saviani, 2008).

Neste artigo tem-se como objetivo analisar a importância da gestão de informação e do conhecimento na implantação da política pública de assistência estudantil, do ponto de vista teórico e algumas propostas de pesquisa nesta linha. É importante destacar que a política pública de assistência estudantil - Pnaes - foi instituída pelo decreto n. 7.234/2010 e pela resolução $n$. 2/2008 e visava a possibilitar à permanência e a conclusão dos cursos pelos estudantes em situação de vulnerabilidade socioeconômica, conforme iremos abordar mais a frente. Recentemente têm existido pesquisas que visam analisar a política pública de assistência estudantil, e como ela realmente se efetiva.

Contudo não se tem dado atenção sobre o papel da gestão da informação e do conhecimento para o aperfeiçoamento da política em questão, visto que o uso da informação e do conhecimento possibilita a alcançar os resultados desejados, pois na medida em que se faz a avaliação da gestão afetiva da política, é necessário associar ao conjunto de estratégias e, neste ponto, a gestão de informação e de conhecimento mostra-se relevante, pois permite o mapeamento dos fluxos formais de informação, a coleta, análise e armazenamento das informações, com objetivo de verificar a efetividade na implantação da política pública da assistência estudantil.

Estudos desenvolvidos por Alves (2002); Araujo (2003); Zago (2006); Portes (2006); Vasconcelos (2011) apresentam um conjunto de informações importantes para compreensão da política, mas enfatizam a análise da política olhando os aspetos relacionados ao ingresso e a permanência de estudantes desfavorecidos economicamente nas universidades públicas. Foi neste sentido que um conjunto de iniciativas, como programas de ação afirmativas em várias universidades foram desenvolvidos, no âmbito dos quais, dentre outras questões, previa a distribuição de bolsas a partir do critério socioeconômico, o que demostra a preocupação do governo em promover o acesso e permanência no ensino superior.

Estas políticas afirmativas são consequência dos diversos estudos produzidos pelo Fórum Nacional de Pró-Reitores de Assuntos Comunitários e Estudantis - Fonaprace -, que revelam que há uma crescente necessidade de implantação de políticas públicas que possibilitem não só o acesso aos estudantes na universidade, como acontece com o Reuni, mas que facilite a permanência do estudante de baixa renda nas instituições federais de ensino superior (Leite, 2014). Este número considerável de estudantes de renda baixa têm crescido, nos últimos anos, devido a criação de ações afirmativas nas universidades destinadas a estudantes egresso da escola pública e estudantes negros (Leite, 2014). 
Neste contexto, notou-se o surgimento das primeiras tentativas de iniciativas de assistência estudantil nas universidades brasileiras, que começam a ocorrer desde os anos 1930, ainda no período do governo de Getúlio Vargas (Lima, 2002; Campos, 2012; Leite, 2012; 2014). Mas foi possível a sua expansão a partir da Constituição Federal de 1988, que nos artigos 205 e 206 que informa que "a educação foi atribuída como dever do Estado e acompanhou os princípios de igualdade de condições de acesso, e permanência na escola". Assim, a assistência estudantil pode ser evidenciada antes da Constituição de 1988, quando ainda integrado na lista do assistencialismo associado à perspectiva do direito (Lima, 2002; Campos, 2012; Leite, 2014). Do ponto de vista de algumas pesquisas que foram realizadas, com destaque a pesquisa de Leite (2014), Campos (2012), Mészáros (2008), Reis (2006), Gazzoto (2016), evidenciam que a assistência estudantil tem a intenção de proporcionar à permanência dos estudantes.

Este artigo apresenta-se como sendo relevante por ser um dos primeiros estudos que se desenvolve no contexto brasileiro e far-se-á analise não apenas da política de assistência estudantil de forma isolada, mas a maneira como deveriam ser utilizadas as informações socioeconômicos dos estudantes para a concessão do auxílio e a gestão das informações e do conhecimento dentro da instituição, com vista ao aperfeiçoamento da implantação da política. Igualmente, este estudo visa a fazer uso de algumas abordagens desenvolvidas no campo das políticas publicas, como é o caso da implantação e avaliação da efetividade e eficácia da política, considerando alguns pressupostos utilizados no campo da ciência e tecnologia, neste caso, a gestão da informação, pois isso vai permitir a produção e análise de indicadores da Ciência dentro da universidade. Finalmente, e não menos importante, este estudo fará uso de diversas estratégias metodológicas, desde a utilização da informação até a análise dos indicadores, isto é, o impacto da política pública na sociedade como um todo, ou seja, procurando verificar se minimizou as desigualdades no acesso ao ensino superior no Brasil.

Este estudo foi exploratório e empírico e utilizou dados primários sobre a importância do uso da gestão de informações e do conhecimento na avaliação das políticas públicas de assistência estudantil. Este estudo se enquadra na lógica do debate a respeito discussão sobre a ciência e sociedade, relacionando a implantação das políticas públicas, olhando a gestão de informação e de conhecimento como um elemento importante na implantação das políticas públicas, e como pressupostos teóricos das políticas públicas fazem uso destes dois elementos - informação e conhecimento - para melhoria na implantação das políticas públicas.

\section{Políticas públicas e política da assistência estudantil: breve discussão teórica}

A discussão sobre as políticas públicas deve ser associada a três pressupostos: o problema em questão, a solução e quem procura solucionar, neste caso o Estado. Para Muller (2000) as políticas públicas ocorrem no momento em que as autoridades, neste caso o governo, reconhecem um problema e buscam resolver ou modificar por meio de um programa ou uma política, seja de ordem cultural, social ou econômica. Nestes termos, o autor entende por políticas públicas os mecanismos que servem como fonte de construção e interpretação de um real problema e definindo modelos normativos de ação 
(Muller, 2000; Carvalho, 2007). Do ponto de vista teórico as políticas públicas fazem parte de um conjunto de estudos das ciências sociais aplicadas ou interdisciplinares que utilizam métodos de pesquisa para analisar as inter-relações entre instituições políticas, processos políticos e o conteúdo politico (Dunn, 1981; Frey, 2000; Capela, 2007).

Conforme Zanardini (2006) o que caracteriza as políticas públicas como campo específico interdisciplinar ligado a ações específicas do Estado, é a preocupação constante de consolidar os projetos da sociedade como um todo. Isto porque o entendimento de que as políticas públicas são longínquas, desenhadas e implantadas por sujeitos concretos e que têm como finalidade a consolidação de um projeto social, político e econômico que normalmente deve refletir as forças internas da sociedade (Zanardini, 2006; Gomide, 2008; Costa, 2010). Em termos gerais, as políticas públicas traduzem, no seu processo de elaboração e implantação, formas diversas de expressão do poder, isto é, se expressam em torno de um processo dinâmico, incluindo negociações, pressões, mobilizações, alianças ou conflito de interesses.

As políticas públicas têm os seus interesses materializados em ações do Estado e na criação de um consenso de que as políticas propostas são eficientes para atender às necessidades de um determinado contexto e de que trarão benefícios para os indivíduos que colaborarem com implantação. Emergem então, as políticas sociais que, na prática não passam de estratégias governamentais de intervenção nas relações de produção e no campo dos serviços sociais (Frey, 2000).

\section{Política pública de assistência estudantil: elementos de complementaridades ou resolução de velhas tendências}

O programa de Reestruturação e Expansão das Universidades Federais - Reuni - foi instituído visando ao processo de ampliação das políticas de inclusão e segundo suas diretrizes pretende congregar esforços para a consolidação de uma política nacional de expansão da educação superior pública. Instituído pelo decreto n. 6.096, de 24 de abril de 2007, o Reuni objetiva a ampliação das vagas nas universidades federais e a redução das taxas de evasão nos cursos presenciais de graduação. Este programa tem como base a meta da "elevação gradual da taxa de conclusão média dos cursos de graduação presenciais para noventa por cento e da relação de alunos de graduação em cursos presenciais por professor para dezoito, ao final de cinco anos, a contar do início de cada plano" (Brasil, 2007).

Além desta meta, cuja racionalização dos gastos públicos é o pressuposto, por meio do aumento do número de concluintes e do número de alunos, por professor, o decreto tem como meta a reorganização dos cursos de graduação pela diversificação das modalidades de graduação e da mobilidade acadêmica. Para Araújo (2011) cria-se condições para a ampliação do acesso e permanência na educação superior, no nível de graduação, pelo melhor aproveitamento da estrutura física e de recursos humanos existentes nas universidades federais. Isto implica na reestruturação física e acadêmica das universidades federais, que propiciará uma expansão no número de vagas na educação superior.

Iniciou-se a partir desse contexto um processo de reflexões e mudanças, em que o acesso e a permanência à educação passaram a ser tratados como direitos. Neste cenário, Araújo (2011) caracteriza que o Reuni, na sua gênese e nos instrumentos utilizados, estabeleceu a ressignificação do conceito de universidade, defendida por 
movimentos ligados à educação, pelos quais as atividades da universidade, de caráter essencialmente público e financiadas pelo Estado, estejam assentadas na dissociabilidade entre ensino, pesquisa e extensão.

A criação do Reuni não resolvia em si o problema do fundo e, por meio disso, foi criada a política pública de assistência estudantil, que faz parte do leque das diversas políticas públicas e sociais existentes com a intenção de mitigar a problemática da equidade e distribuição. A mesma política, vista como sendo um direito estudantil no país, é regulamentada pelo Plano Nacional de Assistência Estudantil - Pnaes -, decreto n. 7.234 de 2010.

Esta política de assistência estudantil é peça importante para possibilitar as condições necessárias para os alunos permanecerem nas universidades, sobretudo os alunos com condições econômicas desfavoráveis. Apesar de a assistência estudantil ser considerada uma estratégia do governo em relação à acessibilidade da educação pública, ela deve ser universal e criar condições para que os desfavorecidos em termos de condições sócio-econômica consigam permanecer até a conclusão do curso (Leite, 2014; Rego e Silva, 2015). Na Universidade Federal de São Carlos, a assistência estudantil é composta por composta pela Bolsa Permanência, Auxílio Moradia e Auxílio Alimentação. Nestes termos, o Pnaes compreende um conjunto de ações necessárias para garantir condições de igualdade no acesso e permanência dos alunos nas instituições de ensino superior.

Nascimento (2012) identifica que é importante problematizar o debate sobre assistência e, por via disso, permitir desenhar o parâmetro da assistência estudantil, associando-o ao conceito de necessidade. O termo assistência, para Sposati (2006) e Yazbek (2006), é relacionado com a oferta de serviços, pois toda política social é um mecanismo de estabilizar as relações sociais, a partir das ações do Estado, assim como de minimizar a subalternização (Yazbek, 2006).

O caráter da assistência é, normalmente, e a modalidade que conforma a política social no Brasil, principalmente com a ação estatal no campo social. De acordo com Sposati et al (1989), é o projeto assistencial que delimita o caráter emergencial às políticas públicas sociais, compreendendo-as como emergência: a capacidade do governo em responder prontamente ou as respostas estatais de forma eventual e fragmentada. Nestes termos, o desenho da política pública no Brasil distancia-se da ideia de universalidade, uniformidade e unificação, que são propostas do Welfare State (Sposati et al, 1989), principalmente no frágil investimento ao longo dos últimos nove anos, como se verifica na tabela 1. 
Tabela 1 -

Orçamento destinado a Política Nacional de Assistência Estudantil (PNAE) - 2008 2017.

\begin{tabular}{c|c|c}
\hline ANO & $\begin{array}{c}\text { Investimento desde 2008 } \\
\text { no PNAE }\end{array}$ & $\begin{array}{c}\text { Taxa de Crescimento do } \\
\text { Orçamento em } \%\end{array}$ \\
\hline 2008 & $\mathrm{R} \$ 125,3$ milhões & $=$ \\
\hline 2009 & $\mathrm{R} \$ 203,8$ milhões & $62,01 \%$ \\
\hline 2010 & $\mathrm{R} \$ 304$ milhões & $49,75 \%$ \\
\hline 2011 & $\mathrm{R} \$ 367$ milhões & $30,0 \%$ \\
\hline 2012 & $\mathrm{R} \$ 504$ milhões & $27,49 \%$ \\
\hline 2013 & $\mathrm{R} \$ 603$ milhões & $19,84 \%$ \\
\hline 2014 & $\mathrm{R} \$ 742$ milhões & $23,01 \%$ \\
\hline 2015 & $\mathrm{R} \$ 995$ milhões & $20,51 \%$ \\
\hline 2016 & $\mathrm{R} \$ 1.030$ Bilhão & $15,08 \%$ \\
\hline 2017 & 987 milhões & $-4,13 \%$ \\
\hline
\end{tabular}

Fonte: MEC (2016; 2017); Câmara dos Deputados Federais.

Verifica-se que, considerando o contexto da expansão e democratização do ensino, que é um dos objetivos do Pnae, houve algum tipo de ampliação de orçamento ao longo dos nove anos, com alguma exceção em 2016. De acordo com a tabela e com os dados da MEC, houve mais de 5 bilhões e meio de reais que foi investido em assistência estudantil desde 2008, no processo de efetivação do Pnaes, até 2017. É importante neste quesito de orçamento considerar o momento político, econômico e social que determina as opções políticas e tipo de políticas a seguir e investir (Nascimento, 2012; Moraes, 2011).

A problemática da construção da política de assistência estudantil passou por um conjunto múltiplo de demandas entre os quais: estudantes ou usuários. No entanto, além disso, há um conjunto de questões que devem ser levantadas: qual público alvo? Quais as necessidades estudantis quem as deve definir? Contudo, o Fonaprace tem sido a principal instituição responsável pela direção da assistência estudantil nos últimos anos, e por isso, necessita ter os dados brutos sobre os estudantes abrangidos pela política e, mais tarde, fazer o uso da informação e de conhecimento que possui para melhor analise no processo de implantação (Nascimento, 2013; Vieira, 2016).

Nascimento (2012) entende que a partir da literatura sobre o debate do assistencialismo das políticas públicas a compreensão que fica é de que a assistência estudantil tem um caráter assistencial da política de educação, isto porque a política pública de assistência estudantil surge a partir das reivindicações em defesa do provimento por parte do Ministério da Educação e o Estado, as condições necessárias à permanência dos estudantes na universidade, pois cabe à assistência social as ações de prevenção e provimento de um conjunto de garantias que cubram, reduzam ou previnam os riscos e vulnerabilidades sociais (Sposati et al, 1989, apud Yazbek, 2004). 


\section{Reflexões sobre gestão de informação e do conhecimento: pontos chaves}

O debate sobre a gestão de informação e do conhecimento é relevante para compreender a implantação das políticas, seja em instituições públicas ou privadas. Entende-se por gestão de conhecimento o conjunto de estratégias para criar, adquirir, compartilhar e utilizar ativos de conhecimento dentro da instituição, bem como estabelecer fluxos que garantem informação necessária no tempo e formato adequados, com vista à geração de ideias, solucionar problemas e tomada de decisão eficazes (Tarapanoff, 2001; Beal, 2004).

Do ponto de vista teórico McGee e Prusak (1994), Davenport e Prusak (1998), Santos (2000), Beuren (2000), Tarapanoff (2001), Beal (2004), avançaram que atividades da gestão da informação e do conhecimento têm em vista transformar os dados existentes em conhecimento. A informação, neste contexto, é uma componente essencial para o desenvolvimento da organização (Barreto, 1994). Sendo assim, o processo de elaboração de informação deve estar direcionada à atribuição de estratégias e mecanismos institucionais que visam a gerar o conhecimento. Barreto (1994) assevera que a informação, quando corretamente utilizada e assimilada, pode produzir conhecimento, tem o poder de modificar o estoque mental de informação do individuo e pode trazer benefícios para o seu desenvolvimento da instituição e da sociedade. Grande parte das instituições públicas, para tomar decisões, deve lidar com uma quantidade de informação com valor agregado, que se apresentam em estado bruto (Gomes e Braga, 2001; Periotto, 2010).

Segundo Gomes e Braga (2001) os processos de gestão de informação e do conhecimento compreendem um conjunto de explicações: informação é utilizada para se referir a todas as descrições de sinais ou dados. Neste contexto, a informação que advém a partir do dado pode ser entendida como significado atribuído a um determinado dado com base no contexto, nas necessidades ou no domínio de um assunto, o que vai possibilitar a tomada de decisão nas atividades humanas. Enquanto o conhecimento é compreendido como informação em sua natureza interpretada, estruturada e aplicada.

Valentim (2002) apresenta os termos quanto a sua natureza e explica que as informações

são aqueles acessados dentro ou fora da organização e podem ser entendidos como aqueles que compõem bancos e bases de dados internos e externos, redes de comunicação como Internet, intranet's, publicações impressas etc. [...] estruturáveis basicamente são aqueles produzidos pelos diversos setores da organização, porém sem seleção, tratamento e acesso. Como exemplo pode-se citar: cartões de visita, colégio invisível, nota fiscal, atendimento ao consumidor, entre outros. [...] não estruturados são aqueles produzidos externamente à organização, porém sem filtragem e tratamento. Alguns exemplos: informações veiculadas na mídia, mais especificamente TV e rádio, boatos, acontecimentos sociais e políticos. (p. 47).

Há outros estudos desenvolvidos por Davenport e Prusak (1998) que exploram os conceitos de informação e conhecimento, assim como a gestão de informação a partir da abordagem ecológica da informação. Os autores consideram estes dois conceitos como uma criação humana e que a sua importância deve ser associada ao papel que os 
mesmos desempenham. Nestes termos, não é fácil distinguir, em termos práticos, a informação do conhecimento.

Neste sentido, ao pensarmos na informação e conhecimento, há uma preocupação com a quantidade de dados disponíveis, que podem ser transformados em informação importante na organização (McGee e Prusak, 1994; Periotto, 2010). Segundo McGee e Prusak (1994), as organizações estão repletas de dados que só serão transformados em informação útil ao tomador de decisão se disponibilizados de maneira que ele possa relacionar e manipulá-los. Isto nos sugere que a informação não se limita apenas aos dados coletados, ela representa os dados coletados, organizados, ordenados, aos quais são atribuídos significados e contextos.

Davenport e Prusak (1998) trazem explicação que foca nas atividades, que enfatiza a transformação dos termos, conforme se vê a seguir. Primeiro, é o nível da informação que é o dado e se referente a itens dispersos e não contextualizados, como, por exemplo, fatos, textos, gráficos, imagens: a principal atividade é a coleta interna ou externa e armazenamento bruto dos dados. A segunda categoria é a informação, caraterizada pela contextualização interpretativa, ou seja, a interpretação individual dentro de um contexto, que inclui o processamento mais refinado dos dados, construindo tabelas, gráficos, exibir coordenadas em um gráfico. A terceira categoria, parte da avaliação dos dados e das informações, que aborda sua confiabilidade, sua relevância e sua importância, para chegar ao conhecimento. Nestes termos, o conhecimento esta envolvido com uma série de conceitos dinâmicos pela interação com o ambiente, o que pode ser chamado de aprendizado.

\section{Importância da gestão de informação e de conhecimento nas instituições de ensino}

A informação é considerada em várias organizações com sendo o fator estrutural e elemento importante para gestão na organização. Dentro de um contexto econômico e social, a função estrutural é considerada como um fator de sucesso para a instituição (Mcgee e Prusak, 1994). Em tempos, verificamos cada vez mais ambientes complexos, no qual a gestão de informação passa ser fundamental para a sobrevivência das organizações e suas atividades. Neste contexto, as organizações e instituições, tal como sucede nas instituições que gerem as assistências estudantis, necessitam de informações, para acompanhar e prover situações do meio ambiente competitivo entre os estudantes; subsidiar estudos básicos do pessoal técnico, visando melhorar os processos e fundamentar investigações e desenvolvimento da política de assistência estudantil; acompanhar inovações tecnológicas, em termos de seu conteúdo e tendências; posicionar-se em termos comparativos em diferentes situações, isto é, pensar o antes e o depois; conhecer oportunidades favoráveis para obtenção e troca de informações entre as instituições de ensino que estão vinculadas a política de assistência estudantil (Fersiva, 1975; Fujino, 1993).

Assim, o objetivo principal da gestão de informação sobre políticas públicas de assistência estudantil deve ser de identificar e potencializar os recursos e a capacidade informacionais de uma organização, bem como ensiná-la a aprender e adaptar-se às mudanças ambientais. De acordo com Davenport e Prusak (1998), a gestão da informação apresenta-se como o conjunto estruturado de atividades que incluem o modo 
como às instituições obtêm, distribuem e usam a informação e o conhecimento. Seguindo a explicação de Hoffmann (2009), a gestão da informação nas instituições de ensino é ponto de partida e tem como finalidade a

agilização dos fluxos informacionais, a integração das atividades e tarefas organizacionais, a otimização dos processos internos e o apoio ao planejamento e a estruturação de sistemas de informações, visando a transformação da informação em conhecimento, por exemplo, utilizando a gestão eletrônica de documentos para facilitar as trocas de informação. (p. 188)

As informações existentes precisam ser gerenciadas da mesma forma que os outros recursos existentes na instituição, razão pela qual é necessário o estabelecimento de políticas e estratégias para organização e tratamento de modo que as informações se apresentem com mais eficácia (Valentim et al., 2003). Na mesma linha, Choo (2003), Duarte, Silva e Costa (2007) explicam que o uso da informação sobre bolsistas e demais estudantes que recebem algum tipo de auxílio das instituições de ensino público deve seguir um conjunto de processos, isto porque, a administração ou gestão de informação deve ser vista como a administração de uma rede de processos que adquirem, criam, organizam e distribuem a informação na instituição. Assim, o autor analisa o uso da informação na instituição em termos de necessidades, e apresenta um modelo processual de gestão de informação, que são: identificação das necessidades da informação sobre assistência estudantil; aquisição da informação; organização e armazenamento da informação; desenvolvimento de produtos e serviços de informação; distribuição de informação; uso da informação.

Em termos de gestão de conhecimento sobre as políticas de assistência estudantil é importante sempre associar a problemática da distinção entre dado, informação e conhecimento, o que vai permitir que não haja uma imprecisão conceitual. Neste sentido, os dados sobre a política de assistência estudantil são tidos como o valor sem significado e são componentes da informação. A informação é o dado com significado e que se apresenta organizado, processado ou estruturado e o conhecimento sobre assistência estudantil seria, neste caso, a informação com valor agregado e um elemento que habilita a tomar decisões, ou seja, é tudo que deve ser conhecido para começar um curso de uma ação. Deste modo, a gestão de conhecimento é plausível e segue cinco estágios, a saber: os dados gerados transformam-se em informação, que se transforma em conhecimento; o conhecimento resulta em ações informadas e estas, por sua vez, produzem resultados satisfatórios na instituição (Murray, 2004).

\section{Considerações finais}

Este artigo teve como objetivo trazer uma discussão teoria e uma proposta de estudo da política da assistência estudantil, focalizando a apresentação de recentes discussões teóricas e empíricas desenvolvidas recentemente sobre a importância da informação e do conhecimento como ferramentas para avaliação da política pública de assistência estudantil.

Neste sentido, o estudo procurou visualizar que para uma análise sobre a importância do uso da informação e do conhecimento é necessário procurar criar uma ligação entre as duas áreas, que são da ciência e da sociedade. Ao mesmo tempo em 
que a produção cientifica visa a solucionar um conjunto de elementos, segue-se que por meio do acúmulo do conhecimento e da informação podem ser encontradas soluções sobre os problemas que carecem a sociedade, este, no entanto, pode ser a assistência estudantil especificamente ou outra.

Em termos gerais, o artigo evidencia que é possível um estudo mais articulado e aprofundado que inclui a gestão de informação e do conhecimento como variável independente na explicação da efetividade e eficácia na implantação da política pública de assistência estudantil como um todo. Com base nas informações disponíveis os atores, neste caso as instituições de gestão da política de assistência estudantil, podem ver os progressos, avanços e retrocessos, o que lhe permitiria análise exaustiva sobre a política pública e seus resultados ou impactos na sociedade.

\section{Referências}

ALVEZ, Elaine Jesus. Políticas sociais de assistência estudantil para estudantes pobres nas universidades públicas. JORNADA INTERNACIONAL DE POLÍTICAS PÚBLICAS, 6 , 2013. Anais ... São Luís: UFMA, 2013.

ARAÚJO, Rhoberta Santana. A implantação do Reuni na Universidade Federal do Pará: um estudo de caso do campus universitário de Altamira. Belém: UFPA, 2011.

BARRETO, Aldo de Albuquerque. A questão da informação. São Paulo em Perspectiva, São Paulo, v. 8, n. 4, 1994, p. 3-8.

BEAL, Adriana. Gestão estratégica da informação: como transformar a informação e a tecnologia da informação em fatores de crescimento e de alto desempenho nas organizações. São Paulo: Atlas, 2004.

BEUREN, Ilse Maria. Gerenciamento da informação: um recurso estratégico no processo de gestão empresarial. São Paulo: Atlas, 2000.

BRASIL. Decreto n. 7234, de 19 de julho de 2010. Institui o Programa Nacional de Assistência Estudantil - Pnaes.

CAMPOS, Denise. Assistência estudantil na UFMT: trajetória e desafios. Cuiabá: UFMT, 2012. 127f. Dissertação (mestrado em Política Social). Universidade Federal de Mato Grosso, Instituto de Ciências Humanas e Sociais, Programa de Pós-Graduação em Política Social.

CAPELLA, Ana. Perspectivas teóricas sobre o processo de formulação de políticas públicas. In: Políticas públicas no Brasil. HOCHMAN, Gilberto et al. (org.). Rio de Janeiro: Fiocruz, 2007, p. 87-124.

$\mathrm{CHOO}$, Chun. A. A organização do conhecimento: como as organizações usam a informação para criar significado, construir conhecimento e tomar decisões. São Paulo: Senac, 2004.

COSTA, Simone Gomes. A equidade na educação superior: uma analise das políticas de assistência estudantil. Porto Alegre: Ufrgs, 2010. 203f. Dissertação (mestrado em Sociologia). Programa de Pós Graduação em Sociologia, Universidade Federal do Rio Grande do Sul.

DAVENPORT, Thomas H; PRUSAK, Laurence. Ecologia da informação: por que só a tecnologia não basta para o sucesso na era da informação. São Paulo: Futura, 1998. 
DAVENPORT, Thomas H. MARCHAND, Donald A. A GC é apenas uma boa gestão da informação? In: DAVENPORT, Thomas $\mathrm{H}$ et al. Dominando a gestão da informação. Porto Alegre: Bookman, 2004 p. 220-232.

DE ASSIS, Anna Carolina Lili; SANABIO, Marcos Tanure; MAGALDI, Carolina Alves, MACHADO, Carla Silva. As políticas de assistência estudantil: experiências comparadas em universidades públicas brasileiras. Revista Gestão Universitária na América Latina GUAL, Florianópolis, 2013, p. 125-146.

DIAS SOBRINHO, José. Educação superior: flexibilização e regulação ou avaliação e sentido público. In: DOURADO, Luiz Fernandes; OLIVEIRA, João Ferreira; CATANI, Afrânio Mendes. Políticas e gestão da educação superior: transformações recentes e debates atuais. São Paulo: Xamã; Goiânia: Alternativa, 2003, p. 97-115.

DO NASCIMENTO, Clara Martins. Elementos conceituais para pensar a política de assistência estudantil na atualidade. Fonaprace - Revista Comemorativa 25 anos: histórias, memórias e múltiplos olhares. Uberlância: UFU, 2012, p. 147-157.

DOS SANTOS, Raimundo Nonato Macedo. Métodos e ferramentas para gestão de inteligência e do conhecimento. Perspectivas em Ciência da Informação, Belo Horizonte, v. 5 , n. 2, 2000, p. 205-215.

DUARTE, Emeide Nóbrega, DA SILVA, Alzira Karla Araujo; DA COSTA, Suzana Queiroga. Gestão de Informação e do conhecimento: práticas de empresa excelente em gestão empresarial extensivas à unidades de informação. Inf. E Soc: Est., João Pessoa, v. 17, n. 1, 2007, p. 97-107.

DUNN, William. Analysis theory, politics and methods. New York: CRC. Press. 2007.

FONAPRACE - FÓRUM NACIONAL DE PRÓ-REITORES DE ASSUNTOS COMUNITÁRIOS E ESTUDANTIS. Plano Nacional de Assistência Estudantil. Brasília: Fonaprece, 2007.

FREY, Klaus. Políticas públicas: um debate conceitual e reflexões referentes à prática da analise de políticas públicas no Brasil. Revista Planejamento e Políticas Públicas. Brasília: Ipea, v. 21, 2000, p. 777-780.

FUGINO, Asa. Serviços de informação tecnológica para empresa industrial: subsídios para planejamento a partir de estudo de usuários. São Paulo: USP, 1993. $145 f$. Dissertação (mestrado em Ciência de Comunicação). Escola de Comunicação e Artes, Universidade de São Paulo.

GAZOTTO, Mireille Alves; GIOMETTI, Analúcia Bueno dos Reis. Assistência estudantil no cenário da Universidade Federal do Triângulo Mineiro - UFTM. GeoGraphos. [En línea]. Alicante. GIECRYAL de la Universidad de Alicante, 2 de junio de 2016.

GOMES, Elizabeth; BRAGA, Fabiane. Inteligência competitiva: como transformar informação em um negócio lucrativo. Rio de Janeiro: Campus, 2001.

HOFFMANN, Wanda Aparecida Moraes. Gestão do conhecimento: desafios de aprender. São Carlos: Compacta, 2009.

KULLMANN, Geila Gonçalves; POZOBON, Luciane Leoratto; DOMINGUES, Renata de Marco; MELLO, Simone Tomazetti. Apoio estudantil: reflexões sobre o ingresso e permanência no ensino superior. Santa Maria: UFSM, 2008.

LEITE, Janete Luzia L. Política de assistência estudantil: direito da carência ou carência de direitos? SER Social, Brasília, v. 14, n. 31, 2012, p. 453-472. 
LIMA, Carla Patrícia Novaes. A arte da participação e a participação pela arte: uma experiência nas Casas de Estudantes Universitárias da UFPE. Serviço Social em Revista, v. 5, n. 1, 2002, p. 47-72.

MCGEE, James; PRUSAK, Laurence. Gerenciamento estratégico da informação: aumente a competitividade e a eficiência de sua empresa utilizando a informação como uma ferramenta estratégica. Rio de Janeiro: Campus, 1994.

MORAES, Michelle Rodrigues de; LIMA, Gleyce Figueiredo. Assistencialização das políticas educacionais brasileiras. Rio de Janeiro: Instituto Federal de Educação, Ciência e Tecnologia do Rio de Janeiro, 2011.

MURRAY, Peter. Como as empresas mais inteligentes obtêm resultados da GC. In: DAVENPORT, Thomas H; MARCHAND, Donald; DICKSON, Tim. Dominando a gestão da informação. Porto Alegre: Bookman, 2004, p. 213-219.

OLIVEIRA, Jo Junia; SARAPU, Paula. Quem paga a conta da cota? Reportagem publicada em 19 de Setembro de 2012 no Jornal Estado de Minas. Belo Horizonte. 2012.

OLIVEIRA, Simone Barros de e VARGAS, Melissa Welter. A Assistência estudantil como espaço privilegiado de educação para os direitos. Fonaprace - Revista Comemorativa 25 anos: histórias, memórias e múltiplos olhares. Uberlância: UFU, 2012, p. 34-46.

RADAELLI, Andressa Benvenutti. Estado e política educacional: Reuni e a expansão do ensino superior público durante o governo Lula. HISTEDBR, v. 11, 2013, p. 27-40.

RÊGO, Ernny Coêlho e DA SILVA, José Ronaldo Ribeiro. Acesso e permanência na educação: os desafios da assistência estudantil. JORNADA INTERNACIONAL DE POLÍTICAS PÚBLICAS, 7, 2015. Anais... São Luís: Universidade Federal do Maranhão, 2015.

SPOSATI, Aldaiza de Oliveira, BONETTI, Dilséa Adeodata, YAZBEK, Maria Carmelita e CARVALHO, Maria do Carmo Brant. Assistência na trajetória das políticas sociais brasileiras: uma questão em análise. São Paulo: Cortez, 1989.

TARAPANOFF, Kira. Informação, conhecimento e inteligência em corporações: relações e complementaridade. In: TARAPANOFF, Kira (org.). Inteligência, informação e conhecimento. Brasília: Ibict/Unesco, 2006, p. 19-36.

VASCONCELOS, Natalia Batista. Programa Nacional de Assistência Estudantil: uma análise da evolução da assistência estudantil ao longo da história da educação superior no Brasil. Revista da Católica, Uberlândia, v. 2, n. 3, 2010, p. 399-411.

VICTOR, Vieira. Universidades federais devem ter corte de até $45 \%$ nos investimentos. Estadão, São Paulo, 11 ago. 2016. Disponível em $<$ http://educacao.estadao.com.br/noticias/geral,alunos-da-medicina-da-usp-entram-emgreve-a-partir-de-segunda,70002080247>. Acesso em 10 set. 2017.

YAZBEK, Maria Carmelita. As ambiguidades da assistência social brasileira após dez anos de Loas. Serviço Social \& Sociedade, n. 77, 2004, p. 11-29.

ZAGO, Nadir. Do acesso à permanência no ensino superior: percursos de estudantes universitários de camadas populares. Revista Brasileira de Educação, v. 11, n. 32, 2006, p. 226-237.

ZANARDINI, João Batista. A ideologia da pós-modernidade e a política de gestão educacional brasileira. Campinas: Unicamp, 2006. 227f. Tese (doutorado em Educação). Faculdade de Educação, Universidade de Campinas. 
Fidel Terenciano é professor na Universidade Católica de Moçambique - FGTI Pemba. Estudante no curso de doutorado em Ciência Política na UFSCar e pesquisador no Centro de Estudos de Partidos Políticos da UFSCar/CNPq - Brasil. Endereço: Rua 7 de Setembro, 1458 - São Carlos - SP - Brasil.

E-mail: fideldeanarosa@gmail.com / fidelt@ufscar.br.

Zito Pedro é professor no Instituto Superior de Gestão, Comércio e Finanças de Moçambique. Estudante no curso de mestrado em Políticas Públicas na Universidade Federal do Rio Grande do Sul.

Endereço: Avenida Alcindo Cacela, 2760 - Belém - PA - Brasil.

E-mail: zitopedro18@gmail.com

António Salião é professor na Universidade Católica de Moçambique - FGTI Pemba. Estudante no curso de doutorado em Economia na Universidade Católica de Porto - Bussines School.

Endereço: Bairro Eduardo Mondlane, 27, Cidade de Pemba - Moçambique.

E-mail: ansaliao@gmail.com/asaliao@ucm.ac.mz.

Recebido em 3 de novembro de 2017.

Aceito 12 de dezembro de 2017. 